\title{
REVISTA DE INVESTIGACIÓN CIENTÍFICA Y TECNOLÓGICA
}

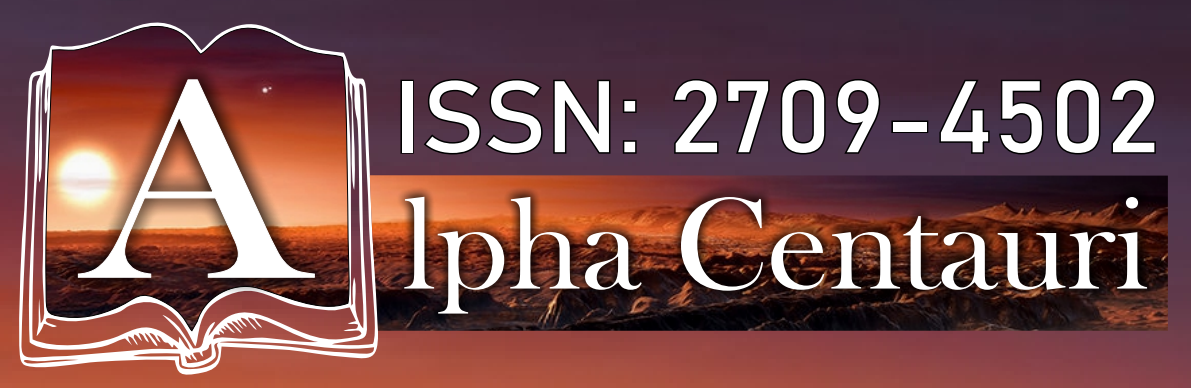

(1)

端要

Energía del viento para aerogeneración:

Una experiencia exitosa

Wind energy for aerogeneration:

A successful experience

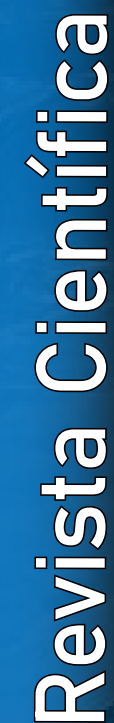

https://do/.org/10.47422/ac.v1i1.5

Todos lo derechos reservados por el grupo Alpha Centauri
Revista Internacional Artículos Científicos Originales 


\title{
Energía del viento para aerogeneración: Una experiencia exitosa Wind energy for aerogeneration: A successful experience
}

\author{
VÉRTIZ OSORES, Jacinto Joaquín \\ Facultad de Ingeniería y Gestión, Escuela de Ingeniería Ambiental \\ Universidad Nacional Tecnológica de Lima Sur \\ RAFAEL-RUTTE, Robert \\ Facultad de Ingeniería y Gestión, Escuela de Ingeniería Ambiental \\ Universidad Nacional Tecnológica de Lima Sur \\ (iD) VÍLCHEZ OCHOA, Guillermo \\ Facultad de Ingeniería y Gestión, Escuela de Ingeniería Ambiental \\ Universidad Nacional Tecnológica de Lima Sur \\ AQUIJE-DAPOZZO, Carmen Luisa \\ Facultad de Ingeniería y Gestión, Escuela de Ingeniería Ambiental \\ Universidad Nacional Tecnológica de Lima Sur \\ CUCHO FLORES, Robert Richard
}

\section{RESUMEN}

Se determinó la energía cinética del viento para aerogeneración como mecanismo de desarrollo limpio (MDL) en base de la velocidad y densidad local del viento durante seis turnos diarios durante tres meses. La velocidad del viento se midió con un anemómetro digital a 3m sobre el relieve terrestre. Se encontró que la energía cinética del viento osciló entre 28.2228252 a 123.48404 J, siendo significativamente menor sólo el mes de abril. No hubo diferencias entre los horarios diarios. Estos resultados representaron $497.79 \%$ a $2177.99 \%$ más que el límite mínimo de generación eléctrica (5.66964 J). Esto se constató con el cálculo de las densidades de potencia del viento: bruta, aprovechable, efectiva y, efectiva con paletas de nanotubos de carbono, encontrándose rangos de 842.21 a $1544.84 \mathrm{~W} / \mathrm{m} 2 ; 499.43$ a $946.09 \mathrm{~W} / \mathrm{m} 2 ; 336.88$ a $617.94 \mathrm{~W} / \mathrm{m} 2$ y 438.00 a $803.30 \mathrm{~W} / \mathrm{m} 2$ respectivamente, proyectadas a 10 y $50 \mathrm{~m}$ para los valores mínimos de velocidad de cada una. Concluyendo que la potencialidad eólica es suficiente para la micro y mediana aerogeneración y califican como MDL. Se recomienda diseñar el modelo matemático del comportamiento de la velocidad del viento relacionado con las alturas de registro para las condiciones climáticas y ambientales de la zona de estudio.

Palabras clave: aerogeneración, límite, generación, potencialidad, eólica, mecanismo. 


\section{ABSTRACT}

Wind kinetic energy for wind power generation as a clean development mechanism (CDM) was determined based on local wind speed and density during six daily shifts over three months. The wind speed was measured with a digital anemometer at $3 \mathrm{~m}$ above the ground. It was found that the kinetic energy of the wind ranged from 28.2228252 to $123.48404 \mathrm{~J}$, being significantly lower only in April. There were no differences between the daily schedules. These results represented $497.79 \%$ to $2177.99 \%$ more than the minimum limit of electricity generation (5.66964 J). This was verified with the calculation of the wind power densities: gross, usable, effective and, effective with carbon nanotube blades, finding ranges from 842.21 to $1544.84 \mathrm{~W} / \mathrm{m} 2$; 499.43 to $946.09 \mathrm{~W} / \mathrm{m} 2 ; 336.88$ to $617.94 \mathrm{~W} / \mathrm{m} 2$ and 438.00 to $803.30 \mathrm{~W} / \mathrm{m} 2$ respectively, projected at 10 and $50 \mathrm{~m}$ for the minimum speed values of each one. Concluding that the wind potential is sufficient for micro and medium aerogeneration and qualify as CDM. It is recommended to design the mathematical model of the wind speed behaviour related to the recording heights for the climatic and environmental conditions of the study area.

Keywords: wind, limit, generation, potentiality, wind, mechanism.

\section{INTRODUCCIÓN}

En nuestro país se tienen que resolver dos problemas: la sustitución del uso de combustibles fósiles que ahonda el problema del cambio climático, convirtiéndonos en un emisor de $\mathrm{CO} 2$, con las características de un país contemplado en la categoría de países del anexo III del Protocolo vigente de Kyoto (FONAMPERÚ, 2007) y, por otra parte, satisfacer la demanda eléctrica de las 41,128 localidades rurales (498,615 viviendas) que posiblemente nunca podrán ser atendidas por el servicio eléctrico convencional (Ramírez, 2006).

La producción de electricidad a través de centrales eólicas es una alternativa para obtener energía eléctrica no contaminante, que evita daños ambientales y que, al compararla con otras formas de producción de energía, tales como las centrales térmicas o el núcleo eléctricas, resulta la más cercana a la sustentabilidad. (Caldera, 2000), sin embargo, para realizar el uso real de la energía eólica se hace necesario contar con mapas eólicos $\mathrm{y}$, para ayudar en el proceso de la formulación de los mapas eólicos a nivel nacional, regional y local, debe procederse a la elaboración de una línea de base. (Jyoti, Painuly, Fenhann, Kamel. \& Pacudan 2005). En ese esfuerzo, Soluciones Solares (2008) publicó el atlas eólico del Perú por regiones en las capitales de los departamentos y algunas ciudades más importantes y accesibles a éstas, los resultados para Huánuco reportan un escaso potencial eólico, pero aún falta precisar muchísimos puntos Eologeográficos del mapa, sobre todo en el área rural, carente de servicio eléctrico público. 
Contribuyendo a esta sinergia de esfuerzos, esta investigación recoge información de línea base fundamentándose en evidencia empírica del trimestre abril - junio, considerado por los pobladores como uno en los que menos fuerza eólica existe en el distrito de Chinchao Huánuco. Partiendo del supuesto que la energía cinética del viento es aprovechable para aerogeneración en esa zona se registró datos de la velocidad del viento usando un anemómetro digital a $3 \mathrm{~m}$ de altura, sirviendo de insumo para los cálculos de la energía cinética. Los resultados obtenidos son muy prometedores para la micro y mediana aerogeneración eléctrica y califican, con suficiencia, para ser consideradas como mecanismos de desarrollo limpio.

Conforme a lo afirmado por Ramos (2009) a partir de un estudio de la Dirección General de Electrificación Rural (DGER) del Ministerio de Energía y Minas - MEM del Perú se menciona que al 2020 aproximadamente 41,128 localidades tal vez nunca podrán ser atendidas por el servicio eléctrico convencional, que representa un $8.27 \%$ del total de comunidades que tienen como única alternativa la electrificación con fuentes energéticas renovables: hidráulica, solar y eólica, sin embargo lejos de recurrir a estas fuentes se prefiere usar combustibles fósiles. Esta política energética no toma en cuenta que el precio del gas natural fluctúa paralelamente con el precio del petróleo cuyos livianos ya están declarados en extinción en nuestro planeta. (Ramos, 2009) además de incrementar los costes de generación.

Gonzales (2007) exploró la energía eólica en zonas rurales encontrando la necesidad de un desarrollo tecnológico y fortalecimiento de aspectos legales para fomentar e intensificar la explotación, así como establecer regulaciones y normas ambientales. La escasez de investigaciones y estudios relacionados sobre el aprovechamiento de la energía cinética de los vientos en nuestro país y en particular en el distrito de Chinchao (Huánuco), impide conocer una línea de base que permita disponer de otras alternativas energéticas.

Medina \& Seccia (s. f.) indicaron:

La energía eólica es la energía cuyo origen proviene del movimiento de masa de aire3 es decir del viento. En la tierra el movimiento de las masas de aire se debe principalmente a la diferencia de presiones existentes en distintos lugares de esta, moviéndose de alta a baja presión, este tipo de viento se llama viento geoestrófico. $(\pi .1)$

Pinilla (1987) señaló que "si la velocidad del viento se duplica, la potencia disponible se incrementa en ocho veces" (p.5). Es por esta razón, que los lugares con altos niveles de velocidad de viento son preferidos para la evaluación sistemática del recurso, así como para la instalación no solo de aerobombas sino también de aerogeneradores de gran tamaño para el suministro de energía eléctrica, aunque esto es más frecuente en países europeos.

González (2007). Planteó que una de las experiencias de investigación e innovación tecnológica en microaerogeneración, constituyó el diseño, construcción y validación de un prototipo de aerogeneración de $500 \mathrm{~W}$ con generador de imanes permanentes realizado en la Universidad Nacional de Ingeniería (UNI), dicho estudio comprendió, el cálculo, diseño y fabricación de cada uno de los componentes del sistema 
aerogenerador: generador de imanes permanentes, rotor eólico y elementos de dirección; así como la evaluación en campo para determinar su performance. Los resultados que obtuvieron fue que la velocidad mínima del viento para microaerogeneración correspondió a $2.8 \mathrm{~m} / \mathrm{s}$.

Chiroque, Sánchez \& Dávila diseñaron microaerogeneradores de baja potencia para aprovechar desde brisas suaves hasta vientos fuertes, fabricados con una combinación de materiales nacionales e importados mediante procesos simples. Estos aerogeneradores estuvieron desarrollados para producir electricidad $\mathrm{y}$ aprovechar un amplio rango de velocidades de viento. Comienzan a generar desde muy bajas velocidades $(3 \mathrm{~m} / \mathrm{s})$ y tienen una curva de generación hasta velocidades de aproximadamente $12 \mathrm{~m} / \mathrm{s}$.

Paredes (2008) identificó en La Libertad, Perú, algunas zonas para la instalación de generadores eólicos, específicamente en Puerto Malabrigo, Paiján, Santiago de Cao y Puerto Salaverry. Para la identificación de estas zonas, previamente se midieron las velocidades del viento, determinándose posteriormente la energía cinética que permitió discriminar las áreas de "exclusión" y las "aptas" durante un periodo mínimo de cinco años. Paredes mencionó además que actualmente, a nivel nacional sólo hay dos aerogeneradores: la Central Eólica Malabrigo (La Libertad), que genera $250 \mathrm{KW}$ y la Central Eólica Marcona (Ica), que genera $450 \mathrm{KW}$.

Soluciones Solares (2009) indicó que existían $4500 \mathrm{MW}$ de concesiones en estudio para generar electricidad con energía del viento en el
Perú y que a inicios de marzo del 2010 estaría listo el mapa eólico del Perú. Asimismo, se estuvo gestando un decreto ley que por primera vez en nuestro país garantizaría un precio de compra y un TIR del 12\% para las energías limpias. Además, Soluciones Solares (2009) precisó que la empresa Ibero Peruana Inversiones aspiraría generar 240 MW a lo largo de la costa, siendo uno de los siete proyectos existentes a la fecha.

En la misma perspectiva de la búsqueda de mayor información de línea base para la aerogeneración, la misma que sería un insumo para futuras aplicaciones tecnológicas de generación energética con desarrollo limpio, se planteó el cuestionamiento sobre la potencialidad de la energía cinética del viento para aerogeneración como mecanismo de desarrollo limpio en el distrito de Chinchao del Departamento de Huánuco durante el mes de mayo del 2009, pudiendo ser un componente importante de las políticas energéticas de la región. Sin embargo, como señalaron Zuluaga, Dyner \& Zapata (2005) además del problema de la poca información técnica generada, la escasez de la difusión de las tecnologías limpias para la generación de la energía -bajo un enfoque costo-beneficio- limita el fomento de la inversión en este rubro, sumándose también, el poco involucramiento de los gobernantes para diseñar una plataforma de financiamiento como: Créditos blandos, certificados y exenciones en impuestos, entre otros. Situaciones que podrían ser abordados en investigaciones ulteriores.

El objetico de la investigación fue determinar la energía cinética del viento para aerogeneración como mecanismo de desarrollo 
limpio en el distrito de Chinchao - Huánuco. Para la consecución de este objetivo general fue necesario determinar la densidad y la velocidad del viento con la finalidad de brindar información precisa, que permita establecer proyectos eólicos de gran envergadura. La finalidad de esta exploración fue definir la viabilidad de la aerogeneración como línea de base, permitiendo referenciar y planificar los estudios al detalle del mapa eólico del distrito de Chinchao.

\section{MATERIAL Y MÉTODOS}

Los materiales utilizados fueron: mapas del distrito de Chinchao; anemómetro marca PCE, modelo PCE-007 con un rango de 0.3 a $45.0 \mathrm{~m} / \mathrm{s}$, resolución de $0.01 \mathrm{~m} / \mathrm{s}$ para caudales volumétricos de 0.061 a 100.0 con una memoria de 2000 registros para trabajar en ambientes de 0 a $80^{\circ} \mathrm{c} \mathrm{y}$ hasta con $80.0 \%$ de humedad relativa; tarjeta de registro de datos multifuncional DAQ, BUSPowered para USB-12-14 bit de $48 \mathrm{~kb} / \mathrm{s}$ con 8 entradas analógicas modelo NI-USB-6009 con sistemas operativos compatibles; software para el registro y procesamiento de la información; reportes climatológicos de la estación del SENAMHI - Huánuco. La investigación fue exploratoria, prolectiva y transversal de una sola medición utilizando dos grupos control (previo y después) correspondiente a los meses de abril y junio, siendo el grupo de interés el mes de Mayo 2009. El universo fue el viento presente sobre el área jurisdiccional del distrito de Chinchao (Huánuco). Los datos se tomaron en el mismo distrito ubicado a $2110 \mathrm{msnm}$, a una latitud de $09^{\circ} 46^{\prime} 45^{\prime \prime}$ y longitud de $76^{\circ} 05^{\prime} 17^{\prime \prime}$.

Los equipos se instalaron usando un poste de madera orientando el anemómetro a la dirección Noreste a una altura de $3 \mathrm{~m}$ sobre el relieve del suelo. El registro fue cada 4 minutos, obteniéndose 10800,11160 y 10800 datos para los meses de abril, mayo y junio del 2009 respectivamente. La información almacenada se agrupó en 6 turnos horarios de cada 4 horas a fin de compatibilizar al comportamiento diario de los parámetros físicoclimáticos registrados por la estación SENAMHI Huánuco (temperatura, presión barométrica y humedad relativa).

Con los datos se procedió a realizar los cálculos de las densidades y, con estos más las velocidades de viento correspondientes, se calcularon las energías cinéticas para cada uno de los 6 periodos horarios establecidos. Con la variable de análisis, se calcularon las densidades de potencia bruta, la densidad de potencia aprovechable-Betz, densidad de potencia efectiva y la densidad de potencia efectiva en aerogeneradores que disponen de paletas de nanotubos de carbono. Para el tratamiento final de datos se realizó un análisis de varianza (ANVA). Para los contrastes significativos se usó la prueba de rango múltiple de Tukey ( $\alpha=5 \%$ ). Finalmente, con la totalidad de los datos de las energías cinéticas de los meses evaluados se procedió a realizar un contraste de una muestra mediante la prueba T Student enfrentándolo al límite mínimo de generación, ( $\mathrm{LmG}$ ) equivalente a $5.6696375 \mathrm{~J}$ (calculado en base a la mínima velocidad de viento 
para aerogeneración: $3 \mathrm{~m} / \mathrm{s}$, reportado por Chiroque, et al. (2008).

\section{RESULTADOS}

Las densidades del viento en los meses de abril a junio 2009 resultaron superiores a $1.225 \mathrm{Kg} / \mathrm{m} 3$ en todo el rango de horarios para el mes de abril. En mayo fue superior con excepción en el rango horario de 8:01 a.m. a $12.00 \mathrm{~m}$. que fue menor $(1.21514 \mathrm{Kg} / \mathrm{m} 3)$ : y en junio fue igual que abril, (Figura 1.)

Figura 1. Densidad del viento $(\mathrm{Kg} / \mathrm{m} 3)$ del viento en los meses de abril a junio de acuerdo al turno horario. Chinchao 2009.

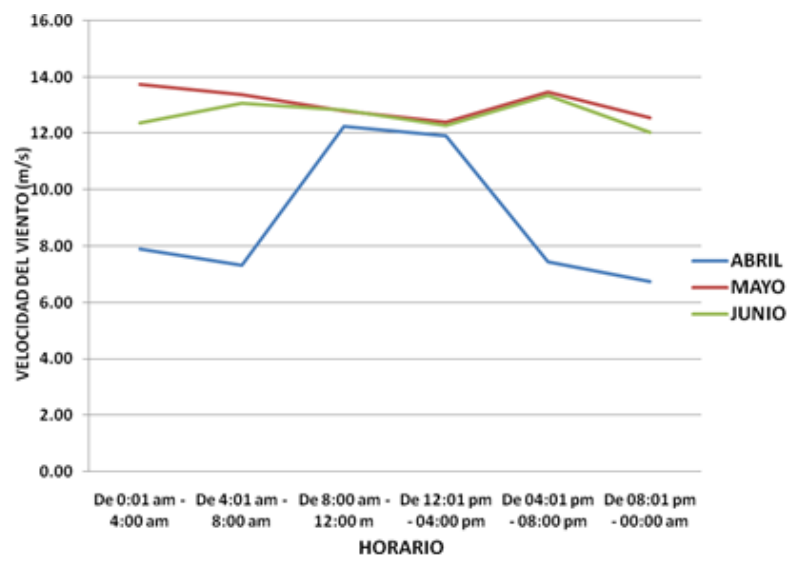

Después del ANVA, no hubo diferencias entre los meses, pero si entre los rangos horarios. Así, desde las 8:01 a.m. hasta las 8:00 pm, la densidad del aire es la misma y desde las 12:01 p.m. hasta las 8:00 a.m. del día siguiente, tampoco es diferente. La mejor densidad del viento se observó en el horario de 8:01 a.m. a 12:00 m. La densidad del viento no se mantiene constante en el distrito de Chinchao.
En el mes de abril se distinguieron las velocidades más bajas del viento con dos picos altos en el horario de 8:00 a.m. hasta las 4:00 p.m. En mayo se mantuvo con mayor uniformidad, siguiendo la tendencia hacia junio. La máxima velocidad del viento fue reportada en mayo con $13.72872 \mathrm{~m} / \mathrm{s}$ correspondiéndole al turno de 0:01 a.m. a 4:00 a.m, mientras que la menor velocidad se observó en abril, en el horario de 08:01 p.m. a 00:00 a.m., con 6.74217 m/s. (Figura 2.).

Figura 2. Velocidad $(\mathrm{m} / \mathrm{s})$ del viento de los meses de abril a junio de acuerdo al turno horario. Chinchao 2009.

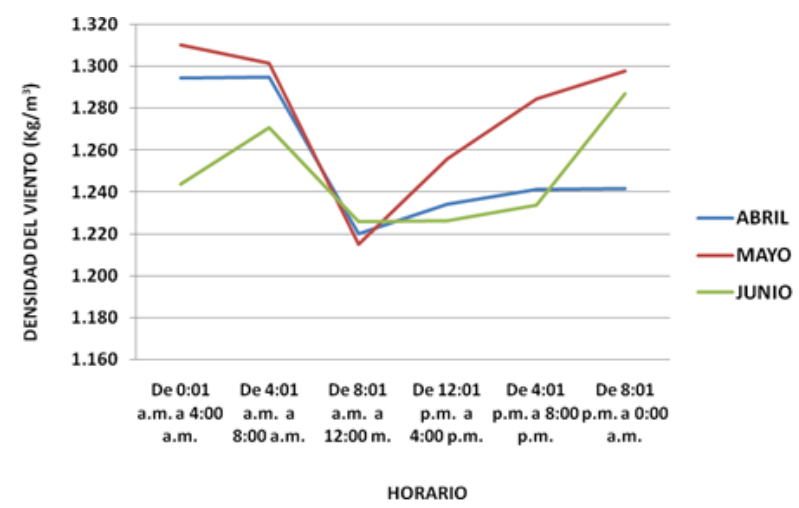

El ANVA indicó que, en promedio, la velocidad del viento es significativa entre los meses de evaluación. La prueba de Tukey indicó que abril fue menor que mayo y junio, no existiendo diferencias entre estos dos últimos. No se encontró diferencias entre los turnos horarios. La energía cinética en abril tuvo los valores más bajos, presentando el mínimo de todos los registros entre las 8:01 p.m. a 0:00 a.m. con 28.2228252 J. En mayo se observó la energía máxima (123.48404 J) entre las 0:01 a.m. a 4:00 a.m., mientras que su mínimo valor (96.5906498 J) significó más del triple del mínimo en abril. En junio el comportamiento fue más uniforme (Figura 3) 
Figura 3. Comportamiento de la Energía Cinética (J) del viento en los meses de abril a junio. Chinchao 2009



El ANVA, indicó que, en promedio, la energía cinética del viento fue diferente sólo entre los meses y no entre los horarios de evaluación. La prueba de Tukey indicó que abril fue significativamente menor que mayo y junio, siendo igual estos dos últimos. Finalmente se realizó la prueba t Student en función al límite mínimo de generación (LmG) obteniéndose que, en promedio, al $95 \%$ de confianza, la energía cinética del viento de los tres meses de evaluación de acuerdo a los seis turnos horarios, fue significativamente superior al LmG

\section{DISCUSIÓN}

Cuesta, Pérez \& Cabrera (2008) refirieron que para cubrir las necesidades eléctricas indispensables para un hogar se necesita $300 \mathrm{KW}$ hora/mes, requiriendo un lugar donde la velocidad del viento sea de $6.26 \mathrm{~m} / \mathrm{s}$. Sin embargo, Chiroque et al. (2008) indicaron que la velocidad mínima para la eologeneración es de $3 \mathrm{~m} / \mathrm{s}$, (parámetro que sirvió para el contraste estadístico de los datos obtenidos en este estudio) y, observando lo reportado por la
UPME (2003), los resultados de la velocidad del viento obtenidos en los meses de evaluación en Chinchao pueden ser viables para aerobombeo y aerogeneración con sistemas autónomos y con sistemas conectados a la red eléctrica, cubriendo suficientemente lo requerido para un hogar.

La energía cinética del viento, fue el insumo para el cálculo de las densidades de potencia que son las que definen la viabilidad de la aerogeneración. Para que el objetivo general de este trabajo evidencie su aplicación, se consideró ampliar los cálculos para llegar a las densidades de potencia (W/m2). Los valores de densidad de potencia obtenidos se basaron en los datos de la potencia bruta obtenida a su vez de las velocidades de viento tomadas a una altura de $3 \mathrm{~m}$, observando que la densidad de potencia más baja se encontraron en abril entre las 8:01 p.m. a 00:00 a.m. (76.11 W/m2) y la máxima fue en mayo entre las 0:01 a.m. a 4:00 a.m. (678.11 W/m2), sin embargo, al proyectarse la velocidad del viento (medido desde $10 \mathrm{~m}$ sobre el relieve del terreno) se tuvo incrementos a medida que aumentó la altura de registro (Asociación Danesa de Industria Eólica, 2003). Tomando como referencia las velocidades de viento hasta de $50 \mathrm{~m}$ de altura de registro, asumiendo una rugosidad de clase 0.5 en la escala de Beaufort (Isemer y Hasse, 1991) y considerando los mismos datos de densidad del viento determinado previamente, se proyectó las densidades de potencia probables para mayo. Así, en el rango más parco de producción de densidad de potencia $(\mathrm{W} / \mathrm{m} 2)$ proyectado de 10 a $50 \mathrm{~m}$ de altura sobre el relieve del terreno para el mes de mayo, osciló entre $336.88 \mathrm{~W} / \mathrm{m} 2$ y $617.94 \mathrm{~W} / \mathrm{m} 2$, 
demostrando así la viabilidad que justifica técnicamente la continuación de las mediciones con mayor detalle para la elaboración del mapa eólico y posterior explotación. Con estas proyecciones aún se tendría amplitud para torres de mayor altura, pues de acuerdo a Gutiérrez, Cazes \& Cataldo (2010) se puede construir torres hasta de $160 \mathrm{~m}$, aunque para este caso quizás la topografía sea una limitante.

Villarubia (2004), manifestó que existe una demanda razonable en la potencia comprendida entre 10 y $100 \mathrm{KW}$ especialmente para conexión a red, pero que requiere disponer de herramientas de diseño similares a las de la tecnología de gran potencia, para lo cual, las empresas basadas en actividad artesanal con desarrollos, primordialmente, de tipo empírico, tienen que evolucionar hacía las nuevas tecnologías que garanticen la fiabilidad y decrezcan el tiempo de desarrollo y, por tanto, el coste final.

Considerando que la población de Chinchao es rural se podría utilizar esta energía eólica para sistemas particulares de generación eléctrica, pudiendo utilizar máquinas eólicas de pequeña potencia, con aplicaciones conectadas a baterías y con capacidad de operar tanto aislados como conectados a red y, así generar empresas rurales para la comercialización de la energía eléctrica tanto para el servicio doméstico como para la instalación de pequeñas industrias que formarían parte de un proyecto ambiental sostenible para este distrito, aunque si se diseña una política energética contextualizada por área geográfica, podría disponerse de generadores de potencia media, aunque tendría que evaluarse en campo las desventajas de pérdida de energía por efecto Joule, como lo sugirió Fernández (2012). Si este escenario energético ocurriría en nuestro país, de todas maneras se necesitaría de una campaña de sensibilización a todos los involucrados, empezando por los miembros de las comunidades universitarias, autoridades, técnicos y sobre todo a los pobladores, ya que como mencionan Simon (1996) y Neira (2006) en países, como el Reino Unido, esta forma de producción de energía es la más aceptada en comparación con cualquier forma de energía no renovable y, la única objeción a este tipo de proyectos es que la población solamente detesta el ruido generado por los aerogeneradores, así como la intrusión paisajística e interferencia electromagnética pero, si las nuevas tecnologías considerasen resolver estas inconveniencias, la aceptación sería total. En esa perspectiva Soren \& Steffen (1999) y, Moragues \& Rapallini (2009) concluyeron que, en términos generales, tanto las poblaciones citadinas como rurales tienden a aceptar proyectos eolo-eléctricos por los beneficios que conllevan y sus mínimos efectos ambientales.

\section{CONCLUSIONES}

La densidad del viento en el distrito de Chinchao fluctuó de 1.21514 a $1.31033 \mathrm{Kg} / \mathrm{m} 3$ en los rangos de horarios estudiados durante los meses de evaluación. Hubo diferencias significativas solamente entre los turnos horarios, Por lo tanto, en nuestras latitudes la densidad del viento es una variable para el cálculo de la energía cinética. 
La velocidad del viento en el Distrito de Chinchao osciló entre 6.74217 a $13.72872 \mathrm{~m} / \mathrm{s}$ en los rangos de horarios estudiados, durante los meses de evaluación. Hubo diferencias significativas sólo entre los tres meses de evaluación, siendo menor solamente abril. Estos resultados representaron el 224.74 a $457.62 \%$ más, de la velocidad mínima requerida para la aerogeneración $(3 \mathrm{~m} / \mathrm{s})$, siendo significativamente mayores. Por lo que la velocidad del viento determinada en la zona de estudio resultó suficientemente apta para aerogeneración.

La energía cinética del viento en el Distrito de Chinchao osciló entre 28.223 a 123.48 J, siendo menor sólo abril. los rangos horarios evaluados fueron iguales Estos resultados fueron significativamente mayores del LmG (5.66964 J), Por lo que la energía cinética del viento determinada en la zona de evaluación resultó prometedora para aerogeneración. Las densidades de potencia del viento bruta, aprovechable, efectiva y efectiva con palas de nanotubos de carbono, se encontraron en los rangos de 842.21 a 1544.84 $\mathrm{W} / \mathrm{m} 2 ; 499.43$ a $946.09 \mathrm{~W} / \mathrm{m} 2 ; 336.88$ a 617.94 $\mathrm{W} / \mathrm{m} 2$ y 438.00 a $803.30 \mathrm{~W} / \mathrm{m} 2$ respectivamente (proyectadas a 10 y $50 \mathrm{~m}$ para los valores mínimos de velocidad de cada una) significando, que la potencialidad eólica de la zona de estudio es suficiente para la micro y mediana aerogeneración eléctrica y califican para ser consideradas como MDL.

Las recomendaciones para otros investigadores es que se trabaje en el diseño del modelo matemático del comportamiento de la velocidad del viento relacionado con las alturas de registro para las condiciones climáticas y ambientales de la zona de estudio. Yendo a la parte aplicativa se debe proponer a la Dirección General de Electrificación Rural (DGER) del Ministerio de Energía y Minas la necesidad de realizar el estudio eólico anual a fin de establecer el mapa eólico para posterior explotación de la industria eólica en la zona de estudio con el propósito de disponer de mayores y mejores alternativas para la generación eléctrica rural en especial en aquellos pueblos donde la oferta del servicio eléctrico convencional es imposible y, no limitarse al uso de los paneles solares que son más costosos que ésta opción. Esta propuesta debe hacerse paralelamente ante los Gobiernos Regionales para el financiamiento de estos estudios y posterior ejecución como proyectos ambientales de inversión.

\section{REFERENCIAS BIBLIOGRÁFICAS}

Asociación Danesa de la Industria Eólica. (2003). Manual de referencia. Conceptos sobre la energía eólica. Publicado el 12 mayo 2003. Recuperado el 18 de mayo de 2019 de http://bit.ly/2d2sZ11

Caldera, M. E. (2000). Potencial de la energía eoloeléctrica en México. México D.F.: Greenpeace.

Chiroque, J., Sánchez, T. \& Dávila C. (2008). Microaerogeneradores de 100 y $500 \mathrm{~W}$. Modelos IT-PE-100 y SP -500. Lima, Perú: Forma e Imagen. 
Cuesta, M. J., Pérez, M. \& Cabrera J. (2008) Aerogeneradores de potencia Inferior a 100 kw. prospectiva y vigilancia tecnológica. Madrid, España: Centro de Investigaciones Energéticas, Medioambientales y Tecnológicas - Ministerio de Ciencia e Innovación.

Fernández, G. (2012). Estudio monográfico sobre tecnologías de generadores eléctricos de media tensión en aerogeneradores. (Trabajo presentado para optar el título de Experto universitario en energía eólica). Universidad Nacional de Educación a Distancia. Madrid. Recuperado de http://bit.ly/2cRTrNJ

FONAMPERÚ (2009). El mecanismo de desarrollo limpio -MDL.: ¿Qué es? ¿Cómo funciona? Recuperado el 12 de Julio de 2019 de http://bit.ly/2de8m4r

Gonzales, S. (2007). Investigación en microaerogeneradores: Implementación de túnel de viento. Lima, Perú: UNI

Gutiérrez, A., Cazes, G. \& Cataldo, J. (2010). Aplicación del modelo WRFARW a la predicción de la generación de energía eléctrica en parques eólicos. Recuperado de http://bit.ly/2drztub

Isemer, H. J. \& Hasse, L. (1991). The Scientific Beaufort Equivalent Scale: Effects on Wind Statistics and Climatological Air-Sea Flux Estimates in the North Atlantic Ocean. J. Climate, 4, 819-836.

Jyoti, P, Painuly, N, Fenhann, J, Kamel, S. \& Pacudan, R. (2005). Wind Power and the CDM. Emerging practices in developing wind power projects for the Clean
Development Mechanism. Energy for Development. Copenhague, Denmark: Ris $\varnothing$ National Laboratory

Medina, C. \& Seccia, P. ( s.f.). Fuentes de generación alternativas. Pontificia Universidad Católica de Chile. Recuperado de: http://bit.ly/2dekF3X

Moragues, J. \& Rapallini, A. (2004). Aspectos ambientales de la energía eólica. Recuperado el 17 de Julio de 2019 de http://bit.ly/2dk215W

Neira, D., Van Den Berg, B. \& De la Torre, F. (2006). El mecanismo de desarrollo limpio en Ecuador: un diagnóstico rápido de los retos y oportunidades en el Mercado de carbono. Quito, Ecuador: Banco Iberoamericano de Desarrollo (BID).

Paredes, R. (2008). Cómo combatir la crisis eléctrica. Centro de Asesoría Industrial de la UPN. Recuperado el 12 de Julio de 2019 de: http://bit.ly/2dikbWy

Pinilla, R. (1987). Energías renovables para el desarrollo sustentable en México. México D.F., México: Secretaría de Energía.

Ramírez, J. (2006). Comentario sobre la ley general de electrificación rural- Ley $\mathrm{N}^{\circ} 28749$. (mayo 30, 2006). Lima Perú.

Ramos, M. (2009). Aspectos generales de la electrificación rural con sistemas fotovoltaico. seminario de energías renovables y medio ambiente en el sector energía región Ucayali. Pucallpa, 28 - 29 de mayo. Perú, Ucayali: Ministerio de Energía y Minas Dirección General de 
Electrificación Rural. Dirección de

Proyectos.

Simon, A. M. A (1996). Summary of Research Conducted into Attitudes to Wind Power from 1990-1996. Londres, UK: Planning and Research for British Wind Energy Association.

Soluciones Solares. Mapa eólico del Perú. (2008). Mapa eólico del Perú. Recuperado el 17 de Julio de 2019 de http://bit.ly/2d2tQPQ

Soren, K. \& Steffen, D. (1999). On Public Attitude Toward Wind Power. Renew Energy 16, 954-957.

Unidad de Planeación Minero Energética - UPME. (2003). Guía para la utilización de la energía eólica para bombeo de agua. Lima, Perú. UPME - Documento No. ANC-603-14.

Villarubia, M. (2004). Energía Eólica. Madrid, España: CEAC

Zuluaga, M. M., Dyner, I. \& Zapata, C. M. (2005). Fuentes alternativas de generación de energía, incentivos y mandatos regulatorios: Una aproximación teórica al caso colombiano. Energética, 55-63.

\section{CORRESPONDENCIA:}

\section{Dr. Jacinto Joaquín Vértiz Osores}

jvertiz@untels.edu.pe 